\title{
Regurgitation Area Segmentation Using the Particle Swarm Optimization and Multilevel Threshold Selection
}

\author{
Kalpana Chauhan ${ }^{1 *}$, M. L. Dewal ${ }^{1}$, Rajeev Kumar Chauhan², Manojkumar Rohit ${ }^{3}$ \\ ${ }^{1}$ Indian Institute of Technology Roorkee, Roorkee 247667, India. \\ 2 Indian Institute of Technology Mandi, Mandi, 175001, India. \\ 3 Post Graduate Institute of Medical Education and Research, Chandigarh 160012, India. \\ * Corresponding author. Tel.: +919410559865; email: kal_2312@rediffmail.com \\ Manuscript submitted July 29, 2014; accepted March 28, 2015. \\ doi: 10.17706/ijcce.2015.4.4.282-289
}

\begin{abstract}
The severity grade of mitral regurgitation is identified easily if the color jet area from the echocardiographic image can be segmented properly. However the echocardiographic images are having many difficulties in the segmentation because there are too many noises, like speckle noise, artifacts and the variations of the human body. The segmentation of the color jet area is a very difficult task. One more problem is that the echocardiographic images obtained from the echocardiographic machines are color images having the color jet inserted on these images. Here we proposed a new technique to solve this problem. There is a combination of two methods viz. Particle swarm optimization (PSO) and multilevel threshold selection (MTS) to separate out the mitral regurgitation jet area.
\end{abstract}

Key words: Mitral regurgitation, echocardiographic, particle swarm optimization, multilevel threshold, segmentation.

\section{Introduction}

Mitral regurgitation (MR) is the insufficiency of the mitral valve and it is very common heart disease in human beings. In case of mitral regurgitation, blood flows backwards through the mitral valve during the contraction of the heart and reduces the amount of the blood that is to be supplied to the body.

Particle swarm optimization (PSO) is a population based research in which each particle is known by its specific position, velocity and off-course from its past data.

Here in this paper the PSO [1] is used in combination with Otsu's multilevel thresholding [2], to segment the MR jet area. An optimum threshold value is selected by finding the maximum value of variance. In some thresholding methods like in [3] the optimal threshold is to be finding out by the maximization of the histogram entropy. In [4] the gray level of each object in the image is assumed to be normally distributed. There are many improvements in the multilevel thresholding time to time [5]-[8].

The hybridization of PSO and multilevel threshoding proposed in this paper overcomes the problem of segmentation of echocardiographic image for the MR severity detection. This hybrid method results in a multilevel segmentation of the echocardiographic images.

\section{Overview of Otsu's Algorithm and Particle Swarm Optimization}

\subsection{Otsu's Multilevel Thresholding Algorithm}


Algorithm for Otsu method is as follows:

Let us assume that the pixels of an image be represented in a number of gray levels say, $L=[1,2, \ldots ., L]$. At some level $j$ the number of pixel may be represented by $n_{j}$. So, the total number of pixels on L level is:

$$
N=\left[n_{1}, n_{2}, \ldots . ., n_{L}\right]
$$

After normalizing the histogram the number of gray levels may be:

$$
p_{j}=n_{j} / N, \quad p_{j} \geq 0, \sum_{j=1}^{L} p_{j}=1
$$

An image may be separated (by a threshold at level $T$ ) from background (with pixels of class $C_{b}$ ) and objective (with pixels of class $C_{o}$ ). $C_{b}$ having the pixels with levels $[1, \ldots, T]$ and $C_{o}$ having the pixels with levels $[T+1, \ldots, L]$. Then the probabilities of the occurrence of two gray levels classes are given by:

$$
\begin{gathered}
\omega_{b}=\sum_{j=1}^{T} p_{j}=\omega(T) \\
\omega_{o}=\sum_{j=T+1}^{L} p_{j}=1-\omega(T)
\end{gathered}
$$

and the class mean levels, are given by

$$
\begin{gathered}
\mu_{b}=\sum_{j=1}^{T} j p_{j} / \omega_{b}=\mu(T) / \omega(T) \\
\mu_{o}=\sum_{j=T+1}^{L} j p_{j} / \omega_{o}=\mu_{L}-\mu(T) / 1-\omega(T)
\end{gathered}
$$

where $\omega(T)$ is the zero ${ }^{\text {th }}$ cumulative moment of the histogram up to the $T^{\text {th }}$ level and can be represented as in eq. (7) and $\mu(T)$ is the first-order cumulative moment of the histogram up to the $T^{\text {th }}$ level and can be represented in eq. (8)

$$
\begin{gathered}
\omega(T)=\sum_{j=1}^{T} p_{j} \\
\mu(T)=\sum_{j=1}^{T} j p_{j}
\end{gathered}
$$

The total mean level $\mu_{L}$ of the original image can be expressed as:

$$
\mu_{L}=\mu(L)=\sum_{j=1}^{L} j p_{j}
$$


The relation can easily be verified for any value of $T$ :

$$
\omega_{b} \mu_{b}+\omega_{o} \mu_{o}=\mu_{L}, \omega_{b}+\omega_{o}=1
$$

The class variances may be given as:

$$
\begin{gathered}
\sigma_{b}^{2}=\sum_{j=1}^{T}\left(j-\mu_{b}\right)^{2} p_{j} / \omega_{b} \\
\sigma_{o}^{2}=\sum_{j=T+1}^{L}\left(j-\mu_{o}\right)^{2} p_{j} / \omega_{o}
\end{gathered}
$$

The with-in-class variance $\sigma_{W}$, between class-variance $\sigma_{B}$ and Total variance $\sigma_{T}$ is given by eq. (13), eq. (14) and eq. (15) respectively.

$$
\begin{gathered}
\sigma_{W}^{2}=\omega_{b} \sigma_{b}^{2}+\omega_{o} \sigma_{o}^{2} \\
\sigma_{B}^{2}=\omega_{b} \omega_{o}\left(\mu_{o}-\mu_{b}\right)^{2} \\
\sigma_{T}^{2}=\sum_{j=1}^{L}\left(j-\mu_{L}\right)^{2} p_{j}
\end{gathered}
$$

The optimal threshold $T$ for bi-level segmentation is maximizes between the class variance as:

$$
\sigma_{B}^{2}\left(T^{\prime}\right)=\max _{1 \leq T<L} \sigma_{B}^{2}(T)
$$

Expression to segment the image on multi-level thresholding can be solved by taking more than one threshold. The image is having $k$ classes for $k$ - 1 thresholds $\left(T_{1}, T_{2}, \ldots, T_{k-1}\right)$. The optimal thresholds can be selected by:

$$
\sigma_{B}^{2}\left(T_{1}^{\prime}, T_{2}{ }^{\prime}, \ldots, T_{k-1}{ }^{\prime}\right)=\max _{1 \leq T<L} \sigma_{B}^{2}\left(T_{1}, T_{2}, \ldots, T_{k-1}\right)
$$

\subsection{Particle Swarm Optimization (PSO)}

The PSO is a population based search in which there is a searching in the space by the particles. The properties of the particles (past value, position and velocity) are characterizing the each particle. All the neighborhood particles follow that particle, which is performing the best (local best) and there is a global best in the entire swarm. The objective function of the particle is to be evaluated during each flight with respect to the particle current position. The information collected from there is used to decide the particle quality so that to determine the leader particle in the sub-swarm or in the complete population of the swarms.

Each individual particle is assumed to be volume-less or we can say assumed to be point in a search space. 
The $i^{\text {th }}$ particle $P_{i}=\left(p_{i 1}, p_{i 2}, \ldots ., p_{i m}\right)$ in the multidimensional search space with a global best particle $p_{g}$. The rate of change in position (velocity) of $i^{\text {th }}$ particle is $V_{i}=\left(v_{i 1}, v_{i 2}, \ldots ., v_{i m}\right)$. The manipulation for the particle can be given as:

$$
\begin{gathered}
v_{i m+1}=v_{i m}+R_{1} \cdot C_{1} \cdot\left(p_{g d}-x_{i m}\right)+R_{2} \cdot C_{2} \cdot\left(p_{i m}-x_{i m}\right) \\
x_{i m+1}=x_{i m}+v_{i m+1}
\end{gathered}
$$

where $C_{1}$ and $C_{2}$ are the positive constants or learning constants, $R_{1}$ and $R_{2}$ are the generated random numbers in the $[0,1]$ interval, $p_{i g}$ is the position of the global best particle and $p_{\text {im }}$ is the position with the best value recorded by the particle so far.

\section{Proposed Hybrid Segmentation Method for MR Jet Area}

The color echocardiographic images are significant for the MR severity evaluation. Assuming that there are $L=(1,2, \ldots, L-1)$ intensity levels for the echocardiographic image. For echocardiographic images the numbers of intensity levels are very large comparison to other types of images. So the Otsu's method alone suffers from the long time processing and decreased efficiency because the number of threshold increases.

So before segment the image through the Otsu's multilevel thresholding, it is to be set with the help of PSO algorithm. With the help of PSO the echocardiographic image has to be converted into the image which having the best levels as mentioned in the eq. (18) and eq. (19).

Let the original echocardiographic image is $I_{E}$. After applying the gbest PSO method there is now only the levels which are very best for the further segmentation process. The obtained image is as follows:

$$
I_{P S O(b l)}=f\left\{P S O\left(I_{E}\right)\right\}
$$

where $I_{P S O(b l)}$ is the image obtained after the application of the PSO gbest formulation. The index term $b l$ represents that the obtained having the best levels which are the features for the further processing.

As we still having the color image with reducing intensity levels, the image has be undergo for Karhunen-Loeve transform (KLT) to find the highest energy component. The low-term components (with highest Eigen values) contain most of the energy In other words the KLT minimizes the geometric mean of the transform component variances. So the image after KLT is as shown below:

$$
I_{K L T}=f\left\{K L T\left(I_{P S O(b l)}\right)\right\}
$$

After the KLT the image is going to the thresholding process given by the Otsu as in eq. (1) to eq. (17). So the segmented image after Otsu's method:

$$
I_{j e t}=f\left\{\operatorname{Otsu}\left(I_{K L T}\right)\right\}
$$

where $I_{j e t}$ represents the jet area segmented image.

\section{Result and Discussions}


For the experiment five cases are considered here. All the five patients are suffering from severe MR. Every case have been discussed in there two views viz. apical 2 or 4-chamber view and parasternal long axis view. The color patterns with jet color representing the MR jet area.

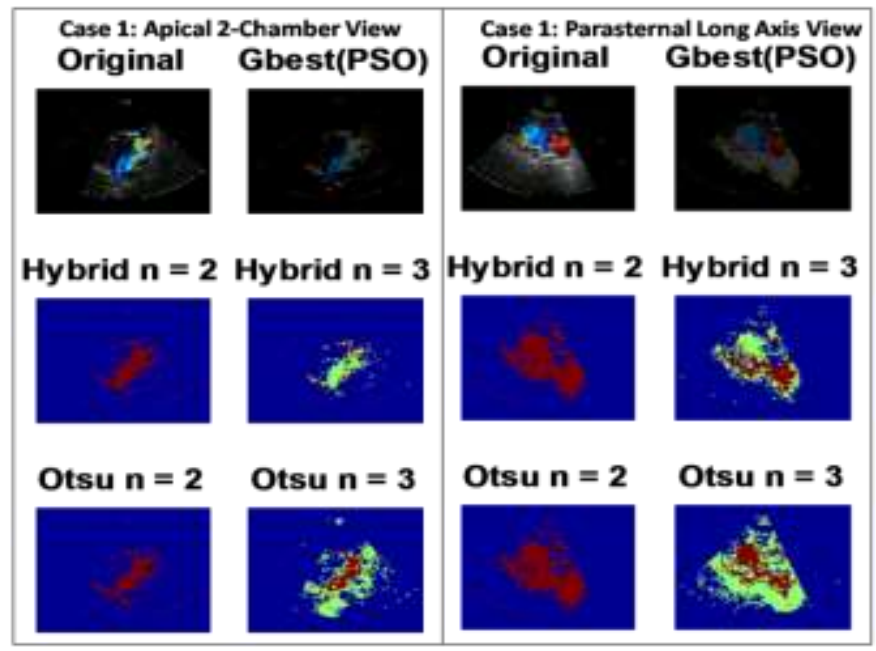

Fig. 1. Case 1 : Apical 2-chamber view and parasternal long axis view.

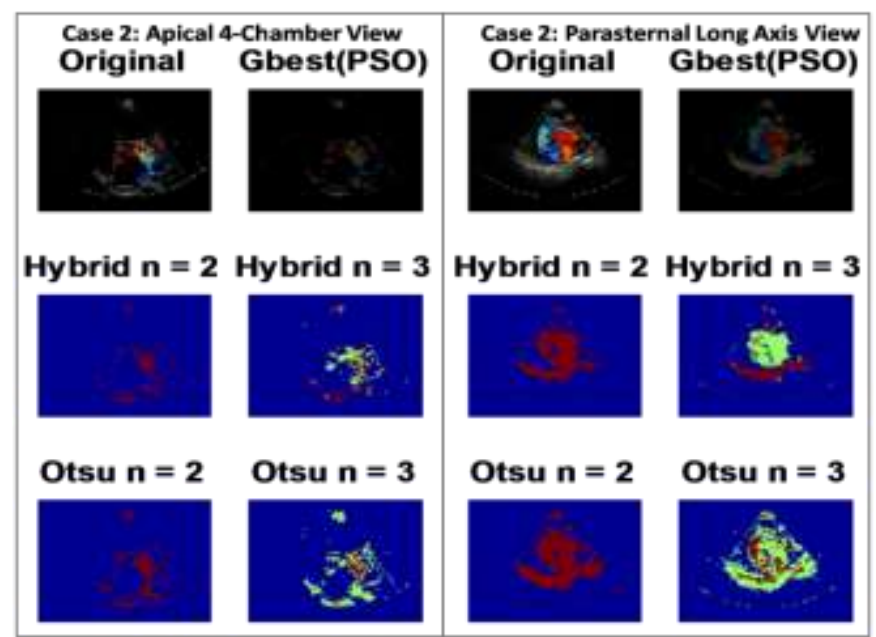

Fig. 2. Case 2: Apical 4-chamber view and parasternal long axis view.

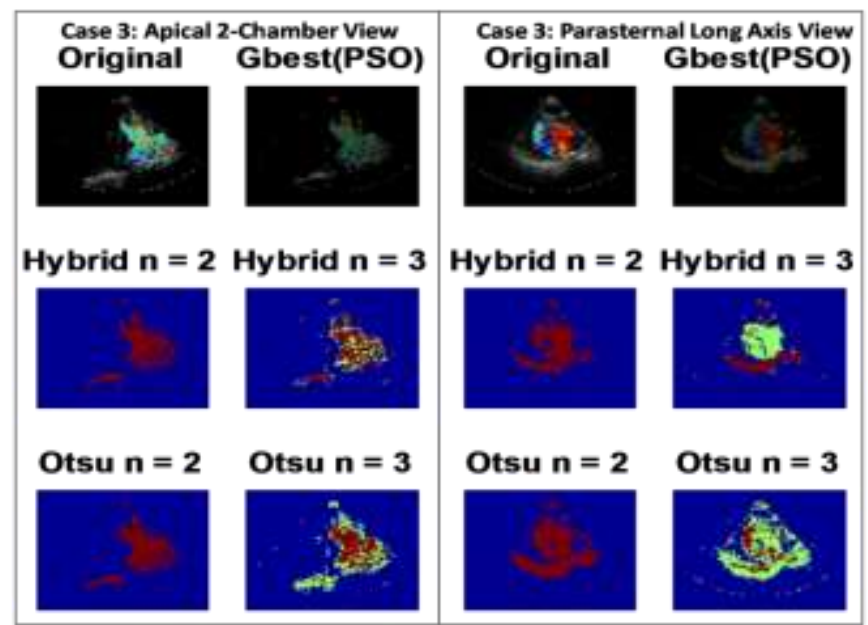

Fig. 3. Case 3: Apical 2-chamber view and parasternal long axis view. 


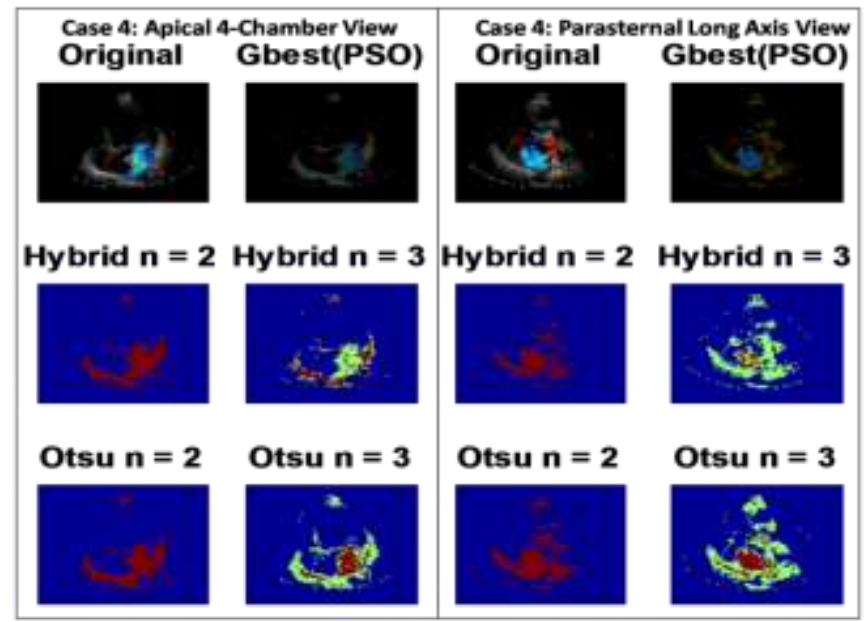

Fig. 4. Case 4: Apical 4-chamber view and parasternal long axis view.

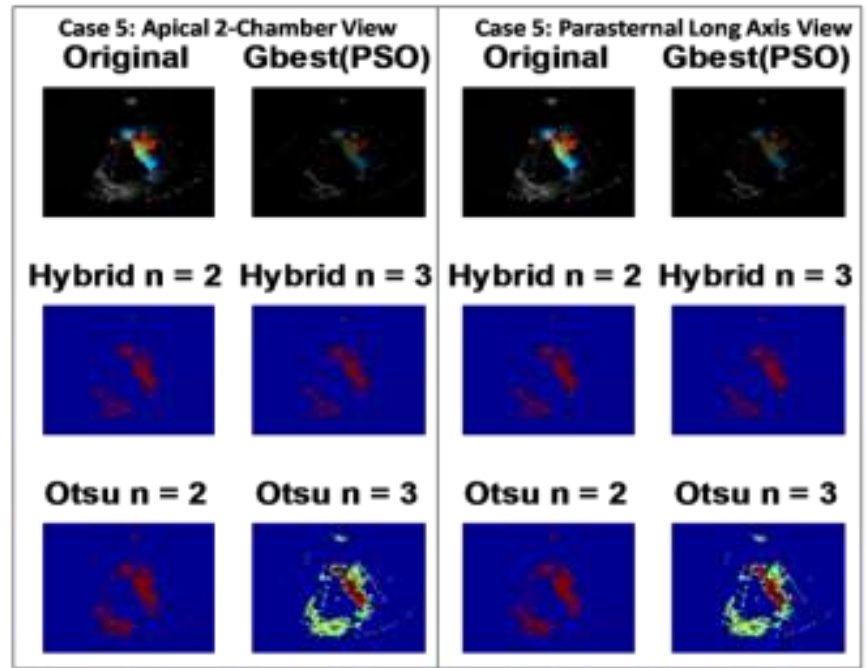

Fig. 5. Case 5: Apical 2-chamber view and parasternal long axis view.

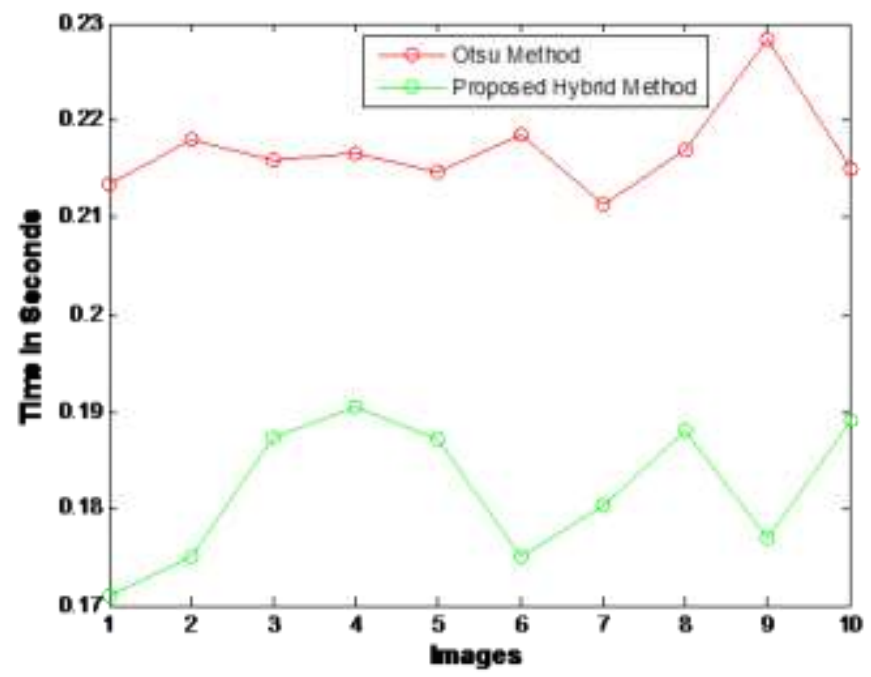

Fig. 6. Graphical representation of the CPU efficiency.

The upper row on the figures showing the original image and the image obtained after the gbest PSO segmentation. It is clear from the image that the intensity levels are decreased in this case. The second and 
third rows are showing the images obtained after applying the proposed hybrid segmentation and after Otsu's segmentation respectively on level 2 and 3.

From Fig. 1 to Fig. 5 it is clear that the segmented images obtained by proposed hybrid method are more centric to MR jet than the images obtained by the Otsu's method alone. However some post-processing are also required for getting the only the MR jet area.

Table 1 showing the comparison of the proposed algorithm with the Otsu's method based on the CPU efficiency. The proposed method takes very less to segment the image. If the image directly be segmented by the Otsu's multilevel thersholding then the convergence time is high. But when it is segmented after the processing by the gbest PSO then the segmentation time is very less as shown in the Table 1 . The time variation can clearly be shown in Fig. 6. The trend line corresponds to the Otsu's method takes the upper area to the trend line corresponds to the proposed method on the time scale.

Table 1. Segmentation Method Comparison Based on CPU Efficiency

\begin{tabular}{|l|l|l|}
\hline Test Image & OTSU'S & Proposed Hybrid \\
\hline Case 1: Apical 2-Chamber View & 0.213487 & 0.171030 \\
\hline Case 1: Paraseternal Long Axis View & 0.217914 & 0.175000 \\
\hline Case 2: Apical 4-Chamber View & 0.215857 & 0.187288 \\
\hline Case 2: Paraseternal Long Axis View & 0.216647 & 0.190480 \\
\hline Case 3: Apical 2-Chamber View & 0.214721 & 0.187042 \\
\hline Case 3: Paraseternal Long Axis View & 0.218538 & 0.175016 \\
\hline Case 4: Apical 4-Chamber View & 0.211351 & 0.180255 \\
\hline Case 4: Paraseternal Long Axis View & 0.216872 & 0.187962 \\
\hline Case 5: Apical 2-Chamber View & 0.228387 & 0.176966 \\
\hline Case 5: Paraseternal Long Axis View & 0.214971 & 0.189045 \\
\hline
\end{tabular}

\section{Conclusion}

In this paper a new method is proposed for the segmentation of the echocardiographic image. The basic propose is to segment the MR jet area echocardiographic images. Otsu's multilevel thresholding is good for such types of image as there are much intensity level are present in the single images. But the method alone takes a large time due to such levels. So the proposed method decreases the level first with the help of PSO gbest method and then Otsu's method is applied. The combination of two methods increases the stability.

\section{References}

[1] Ghamisi, P., Couceiro, M. S., Benediktsson, J. A., \& Ferreira, N. M. F. (2012). An efficient method for segmentation of images based on fractional calculus and natural selection. Expert Systems with Applications, 39, 12407-12417.

[2] Otsu, N. (1979). A threshold selection method from gray-level histograms. IEEE Trans. Syst. Man Cybern., 9, 62-66.

[3] Kapur, J. N., Saho, P. K., \& Wong, A. K. C. (1985). A new method for gray-level picture thresholding using the entropy of the histogram. Computer Vision, Graphics and Image Processing, 29, 273-285.

[4] Kittler, J., \& Illingworth, J. (1986). Minimum error thresholding. Pattern Recognition, 19, 41-47.

[5] Tsai, D.-M. (1995). A fast thresholding selection procedure for multimodel and unimodel histograms. Pattern Recognition, 16, 653-666.

[6] Yin, P.-Y. (1999). A fast scheme for multilevel thresholding using genetic algorithms. Signal Processing, 72, 85-95. 
[7] Fogel, D. B. (2000). Evolutionary Computation: Toward a New Philosophy of Machine Intelligence (2nd ed.). Piscataway, NJ: IEEE Press.

[8] Eberhart, R. C., \& Kennedy, J. (1995). A new optimizer using particle swarm theory. Proceedings of the Sixth International Symposium on Micro Machine and Human Science (pp. 39-43).

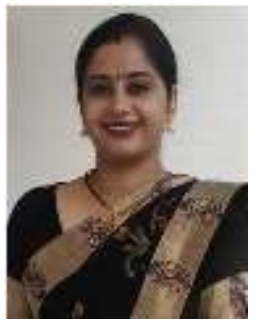

Kalapna Saini graduated from the Department of Electrical and Electronics, College of Engineering Roorkee, Roorkee India, in 2006 and received her master's degree in instrumentation and control engineering from Dr. B. R. Ambedkar National Instiute of Technology Jalandhar, Punjab, India in 2009. She is currently a research scholar with M \& I Group of the Department of Electrical Engineering at IIT Roorkee, Roorkee, India. Her current research interest includes medical image processing. She had published some research papers in different areas. She had attended some conferences, courses training programs and workshops. She is a member of some professional bodies also. Her employment experience includes the University of Texas at Austin, USA.

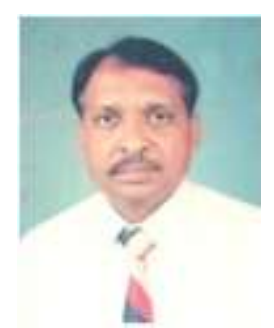

M. L. Dewal received his B.Tech degree in electrical engineering in 1972 from G.B. Pant University of Agriculture and Technology, Pantnagar, India. He did his M.E. (PSE) and Ph.D. degrees in electrical engineering both from IIT, Roorkee, erstwhile University of Roorkee in the year 1974 and 1982, respectively. Currently he is working as a professor in IIT Roorkee. His areas of interest are power system protection and digital image processing.

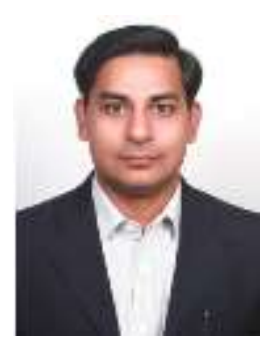

Rajeev Kumar Chauhan graduated in electrical engineering from Institutions of Engineers (India). He received his M.Tech degree in control and instrumentation engineering from Dr. B. R. Ambedkar National Institute of Technology Jalandhar, India. Presently, he is pursuing his Ph.D. degree from the School of Computing and Electrical Engineering, Indian Institute of Technology Mandi, India. His employment experience includes the University of Texas at Austin, USA, the Krishna Engineering College (KEC) GZB, Galgotias College of Engineering and Technology (GCET) Greater Noida, Roorkee Institute of Technology (RIT) Rookee. His major field of interest included DC Microgrid, SCADA System, industrial automation and control. He is a member of IET, IAENG Hong Kong and IACSIT.

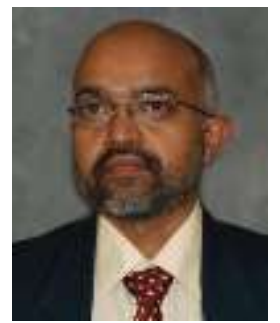

Manojkumar Rohit has done his M.B.B.S. degree from Jawaharlal Institute of Post Graduate Medical Education \& Research-Pondicherry in April 1992. After that he has done his M.D. degree from AIIMS Delhi in May 1996. He worked as a senior resident in AIIMS during Oct 1996-March 1998 and as a D.M. (Cardiology) in PGIMER Chandigarh up to May 2000. Currently he is working as an additional professor in Advanced Cardiac Center in PGIMER Chandigarh since July 2007. 